\title{
Spontaneous Time-Reversal Symmetry Breaking at Individual Grain Boundaries in Graphene
}

\author{
Kimberly Hsieh $\odot,{ }^{1, *}$ Vidya Kochat, ${ }^{1, \dagger}$ Tathagata Biswas, ${ }^{1, *}$ Chandra Sekhar Tiwary, ${ }^{2, \S}$ Abhishek Mishra, ${ }^{3}$ \\ Gopalakrishnan Ramalingam, ${ }^{4}$ Aditya Jayaraman, ${ }^{1}$ Kamanio Chattopadhyay, ${ }^{2}$ \\ Srinivasan Raghavan, ${ }^{3,4}$ Manish Jain, ${ }^{1}$ and Arindam Ghosh ${ }^{1,3}$ \\ ${ }^{1}$ Department of Physics, Indian Institute of Science, Bangalore 560 012, India \\ ${ }^{2}$ Department of Materials Engineering, Indian Institute of Science, Bangalore 560 012, India \\ ${ }^{3}$ Centre for Nano Science and Engineering, Indian Institute of Science, Bangalore 560 012, India \\ ${ }^{4}$ Materials Research Center, Indian Institute of Science, Bangalore 560 012, India
}

(Received 30 September 2020; accepted 30 March 2021; published 19 May 2021)

\begin{abstract}
Graphene grain boundaries (GBs) have attracted interest for their ability to host nearly dispersionless electronic bands and magnetic instabilities. Here, we employ quantum transport and universal conductance fluctuation measurements to experimentally demonstrate a spontaneous breaking of time-reversal symmetry across individual GBs of chemical vapor deposited graphene. While quantum transport across the GBs indicate spin-scattering-induced dephasing and hence formation of local magnetic moments, below $T \lesssim 4 \mathrm{~K}$ we observe complete lifting of time-reversal symmetry at high carrier densities $\left(n \gtrsim 5 \times 10^{12} \mathrm{~cm}^{-2}\right)$ and low temperature $(T \lesssim 2 \mathrm{~K})$. An unprecedented thirtyfold reduction in the universal conductance fluctuation magnitude with increasing doping density further supports the possibility of an emergent frozen magnetic state at the GBs. Our experimental results suggest that realistic GBs of graphene can be a promising resource for new electronic phases and spin-based applications.
\end{abstract}

DOI: 10.1103/PhysRevLett.126.206803

Structural disorder in graphene originates from defects classifiable into two categories: point defects (vacancies, Stone-Wales defects) and extended defects such as grain boundaries (GBs). Vacancies result in localized states close to zero energy leading to magnetic moment formation in graphene, experimentally confirmed by the observation of spin-split resonances in scanning tunneling microscopy (STM) at monovacancies [1], measurements of spin currents [2], and the possibility of the Kondo effect [3,4]. GBs lead to local modification of graphene band structure by introducing weakly dispersing, nearly flat electronic bands with enhanced density of states (DOS), either at zero energy [translational $\mathrm{GB}(2,0) \mid(2,0)][5,6]$ or finite energies [tilt $\mathrm{GB}(5,0) \mid(3,3)][7-9]$. While preliminary studies projected GBs as detrimental to electronic transport [10-17], successive experiments showed that these drawbacks can be overcome by tailoring the growth conditions $[18,19]$. Magnetotransport measurements across isolated GBs reveal enhanced weak localization (WL) compared to single-crystalline grains, indicating stronger intervalley carrier scattering due to lattice disorder $[10,17]$. However, a comprehensive study of the symmetry-breaking mechanisms at graphene GBs through direct measurements of the universal conductance fluctuations (UCF) in the inter- and intragrain regions has so far been lacking.

Magnetic ordering has been predicted at GBs, either by localization at nontrivially coordinated C-rings [7] or assisted by strain, e.g., in translational line defects with octagon-pentagon pairs [5,6]. In realistic GBs realized during chemical vapor deposition (CVD) growth, nucleation centers grow independently and fuse in the local bonding environment. Such GBs comprise multiple defect realizations, including vacancies, Stone-Wales defects, intermittent $1 \mathrm{D}$ line defects [12,20], multimembered C-rings [7,21], etc., causing a strong increase in charge carrier scattering and electronic noise $[14,18,19]$. Despite both numerical [5-7] and spectroscopic $[8,9,20,22]$ evidences of large enhancement in local DOS and spin splitting, no tangible impact of $e-e$ interaction at the GBs has so far been observed. This work combines quantum transport and UCF to probe local charge and spin excitations across individual graphene GBs. The UCF magnitude, determined by the symmetry of the underlying Hamiltonian via the Wigner-Dyson parameter $\beta$ [23], reveals a full spontaneous lifting of the time-reversal symmetry (TRS) in the GB region for $T \lesssim 2 \mathrm{~K}$ and $n \gtrsim 5 \times 10^{12} \mathrm{~cm}^{-2}$. The temperature and density dependence of UCF link the TRS lifting to a frozen magnetic state arising from the GB defect sites.

We measured three devices (D1, D2, and D3) from CVDsynthesized graphene [see the Supplemental Material (SM) [24], Sec. S1], optimized to ensure partial fusion of crystallites [scanning electron micrograph in Fig. 1(a)]. High-resolution transmission electron microscopy (HRTEM) [Fig. 1(b)] performed on a pair of similarly synthesized grains reveals 

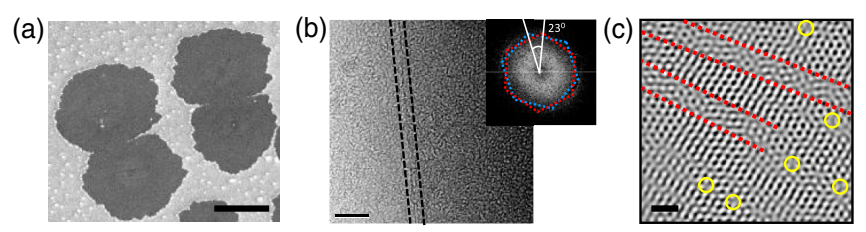

FIG. 1. (a) Scanning electron micrograph of a typical pair of graphene grains with grain size $\approx 15 \mu \mathrm{m}$ forming a GB in between. Scale bar, $10 \mu \mathrm{m}$ (b) Bright field TEM image of the GB formed between two grains. The selected area electron diffraction pattern in the inset shows the misorientation angle between the grains $\approx 23^{\circ}$. Scale bar, $5 \mathrm{~nm}$. (c) HRTEM image of the GB region where line and point defects are outlined. Scale bar, $1 \mathrm{~nm}$.

an average width $\sim 10 \mathrm{~nm}$ of the disordered region and a misorientation angle $\approx 23^{\circ}$ between the parent crystallites. The GBs form a highly disordered region consisting of arrays of line dislocations and undercoordinated $\mathrm{C}$ atoms [Fig. 1(c)] similar to that observed in STM and TEM studies $[8,9,19,20,22]$.

Figure 2(a) schematically describes the conceptual basis of our experimental approach. The quantum interference effect that underpins both quantum corrections to conductivity $\left(\Delta \sigma_{c}\right)$ and the UCF $\left(\left\langle\delta G_{\phi}^{2}\right\rangle\right)$ depends on crossings of time-reversed path pairs as the electron (or hole) diffuses across the sample over $\tau_{D}=L^{2} / D$, the Thouless time, where $L$ and $D$ are the length of the system and carrier diffusivity, respectively. While $\Delta \sigma_{c}$ is determined by the probability of single self-crossing, the correlation function in $\left\langle\delta G_{\phi}^{2}\right\rangle \sim\langle G(0) G(\tau)\rangle_{\tau}$ requires two spatially separated crossing points (thus involving a larger number of defect sites), thereby defining closed loops encircled either in the same (diffusons) or opposite (cooperons) senses with identical structure factors [Figs. 2(a1) and 2(a2)]. This has two important consequences: first, compared to $\Delta \sigma_{c}$ $\left[\sim \ln \left(\tau_{D} \gamma\right)\right]$, the UCF magnitude $\left\langle\delta G_{\phi}^{2}\right\rangle \sim\left(\tau_{D} \gamma\right)^{-2}$ is exponentially more sensitive to emergent dephasing processes in two dimensions, where $\gamma$ is the dephasing rate and thus a more suitable tool when the dephasing processes are confined within spatially restricted regions such as the GBs. Second, when TRS is lifted, usually by a transverse magnetic field $B \gg B_{\phi}, B_{\phi}$ being the field corresponding to one flux quantum threading a phase coherent cell, the cooperon contribution is removed, decreasing $\left\langle\delta G_{\phi}^{2}\right\rangle$ exactly by a factor of 2 . The reduction factor is protected by the symmetry of the underlying Hamiltonian, i.e., $\left\langle\delta G_{\phi}^{2}\right\rangle \sim$ $\left(e^{2} / h\right)^{2} / \beta$, where $\beta=1$ for time-reversal-invariant systems (orthogonal ensemble) and $\beta=2$ when TRS is absent (unitary ensemble). For a time-reversal-invariant system [35], a crossover function $\nu(B)$ is defined as

$$
\nu(B)=\frac{N(B)}{N_{\phi}}=1+\frac{2}{b^{2}} \sum_{n=0}^{\infty} \frac{1}{\left[\left(n+\frac{1}{2}\right)+\frac{1}{b}\right]^{3}},
$$

(a1)

(a2)

(a3)

(b)
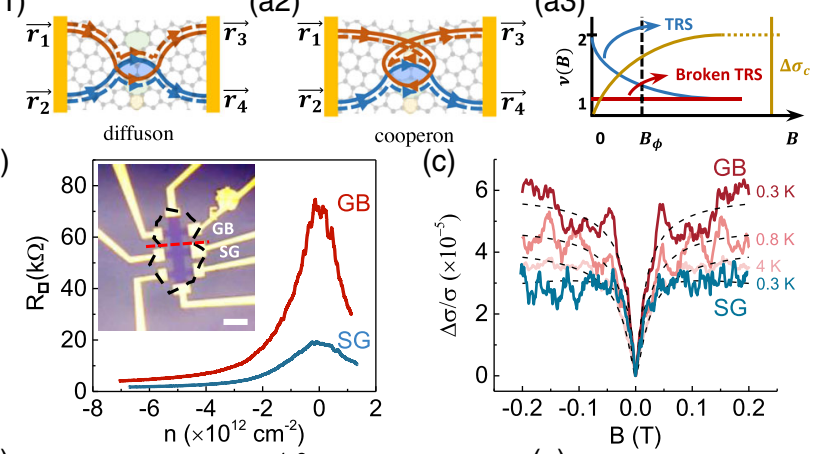

(c)

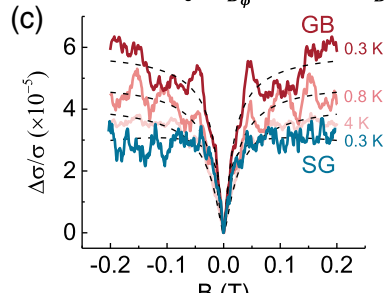

(d)
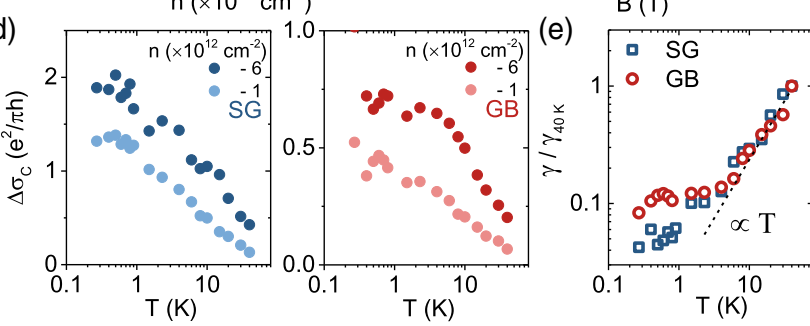

FIG. 2. (a) Schematic showing (a1), a pair of crossings representing diffusons; (a2), a pair of crossings representing cooperons; and (a3), the expected behavior of $\nu(B)$ as a function of $B$ for TRSinvariant and TRS-broken systems. (b) Sheet resistance $\left(R_{\square}\right)$ as a function of gate voltage $\left(V_{\mathrm{BG}}\right)$ for intragrain $(\mathrm{SG})$ and intergrain (GB) regions of D1 at $T=0.3 \mathrm{~K}$. Inset: Optical micrograph of a typical device. The morphology of the original pair of coalesced grains is shown (black line) along with the approximate GB location (red line). Scale bar, $10 \mu \mathrm{m}$. (c) Magnetoconductance measurements are shown for $n=-6 \times 10^{12} \mathrm{~cm}^{-2}$ at $T=0.3 \mathrm{~K}$ for the SG region and $T=0.3,0.8$, and $4 \mathrm{~K}$ for the GB region, clearly exhibiting WL. Dashed lines correspond to HLN fits. (d) Quantum correction to conductivity $\Delta \sigma_{c}$ in units of $e^{2} / \pi h$ plotted for both SG and GB regions as a function of $T$ for $n=-1 \times 10^{12} \mathrm{~cm}^{-2}$ and $-6 \times 10^{12} \mathrm{~cm}^{-2}$. (e) Scattering rate $\gamma$ normalized to its value $\gamma_{40 \mathrm{~K}}$ at $T=40 \mathrm{~K}$, plotted versus $T$ for the SG and GB regions at $n=-6 \times 10^{12} \mathrm{~cm}^{-2}$, is shown, where the black dotted line indicates the temperature regime where Nyquist scattering dominates.

where $b=8 \pi B\left(l_{\phi}\right)^{2} /(h / e)$ is the dimensionless magnetic field that captures the reduction in the UCF as a function of $B$ [Fig. 2(a3)]. Here, $N(B)$ and $N_{\phi}$ are the values of $\left\langle\delta G_{\phi}^{2}\right\rangle$ at $B$ and at $B \gg B_{\phi}$, respectively. When TRS is spontaneously removed (magnetic systems), $\nu(B)$ remains unaffected at the scale of $B_{\phi}$, as observed in ferromagnetic films [36].

For electrical transport, the graphene grains were transferred on to $\mathrm{Si} / \mathrm{SiO}_{2}$ substrates, patterned into Hall bars such that measurements across the GB and within the single-crystalline intragrain (SG) region can be carried out simultaneously [Fig. 2(b) inset]. The excess disorder in the GB region results in enhancement in the resistivity by a factor of $\sim 2$ to 5 times that of the SG region depending on $n$ [Fig. 2(b)] and a consequent suppression of the carrier mobility $\left(\mu_{\mathrm{SG}} \approx 480 \mathrm{~cm}^{2} \mathrm{~V}^{-1} \mathrm{~s}^{-1}\right.$ while $\left.\mu_{\mathrm{GB}} \approx 220 \mathrm{~cm}^{2} \mathrm{~V}^{-1} \mathrm{~s}^{-1}\right)$. Magnetotransport measurements 
down to $T=0.3 \mathrm{~K}$ for a fixed $n\left(\approx-6 \times 10^{12} \mathrm{~cm}^{-2}\right)$ indicate enhanced WL correction at the GB region [Fig. 2(c)], signifying stronger intervalley scattering from short range lattice defects $[10,17,37]$. Fitting [dashed lines in Fig. 2(c)] the modified Hikami-Larkin-Nagaoka (HLN) expression for graphene [38] to magnetoconductance yields both the quantum correction to conductivity $\Delta \sigma_{c}$ and the dephasing length $l_{\phi}$ [Fig. S2(a)]. The $T$ dependence of $\Delta \sigma_{c}$ in Fig. 2(d) shows that the quantum correction behaves differently between SG (left) and GB (right) regions, especially at high $n$. In both cases, we find $\Delta \sigma_{c} \propto \ln (T)$ at low $n$, as expected for diffusive nonmagnetic conductors where dephasing takes place via Nyquist scattering from $e$-e interaction so that $\gamma=D / l_{\phi}^{2} \propto T$. Direct evaluation of $\gamma$ [Figs. 2(e) and S2(b)] from HLN fits confirms this proportionality with $T$. The quantum correction in the GB region at high $n\left(\gtrsim 5 \times 10^{12} \mathrm{~cm}^{-2}\right)$, however, deviates from this behavior, where we find both $\Delta \sigma_{c}$ [Fig. 2(d), right panel] and $\gamma$ [Fig. 2(e)] saturate below $T \sim 7 \mathrm{~K}$.

Low temperature saturation of $\gamma$ in metals is often attributed to inelastic processes from spin-flip scattering [39-41]. Neglecting electron-phonon scattering at such temperatures [42,43], we can write $\gamma=\gamma_{e e}+\gamma_{s}$, where $\gamma_{e e}$ is the $e-e$ induced scattering rate and $\gamma_{s}$ is the spin-flip scattering rate due to dilute magnetic impurities. The observed saturation in $\gamma$ at temperatures $T \lesssim 7 \mathrm{~K}$ can thus be explained from increasing Nyquist scattering $\left(\gamma_{e e} \propto T\right)$ countering the reduction in $\gamma_{s}$ above the Kondo temperature $T_{\mathrm{K}}[44,45]$. Such an anomalous $T$ dependence of $\gamma$ in the intergrain region hints at the formation of magnetic moments that can interact at lower temperatures leading to frozen magnetic ordering [46]. However, the competing effects of localization and antilocalization due to graphene's chiral charge carriers makes it ambiguous to detect or claim such possibilities using WL alone.

To complement quantum transport, we carried out UCF measurements in two different ways: (1) From slow timedependent fluctuations in the conductance relating directly to the ensemble fluctuations of disorder configuration via ergodic hypothesis [35,47-49] (Fig. 3, SM Sec. S4), and (2) by analyzing the reproducible and aperiodic fluctuations in $G$ by tuning the Fermi energy [Fig. 4(b), SM Sec. S5). The time-dependent conductance fluctuations across the GB of D1 at $n=-0.8 \times 10^{12} \mathrm{~cm}^{-2}$ is plotted in Fig. 3(a), clearly displaying a reduction in the relative magnitude of fluctuations at $B=0 \mathrm{~T}$ and $B=47 \mathrm{mT}$ $\left(\gg B_{\phi}\right)$. Figure $3(\mathrm{~b})$ shows the $B$ dependence of $\nu(B)$, defined in Eq. (1), from $\left\langle\delta G_{\phi}^{2}\right\rangle$ evaluated from timedependent conductance fluctuations in device D1 for three different $n$ at the GB region. At low $n\left(\approx 0.8 \times 10^{12} \mathrm{~cm}^{-2}\right)$, $\nu(B)$ shows a clear factor-of-2 reduction as $B$ increases beyond $\sim 30 \mathrm{mT}$, which corresponds to $B_{\phi}$ [Fig. 3(b), uppermost panel]. This suggests TRS to be preserved in (a)

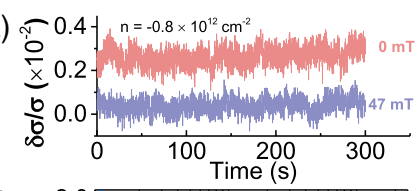

(b)

(c)
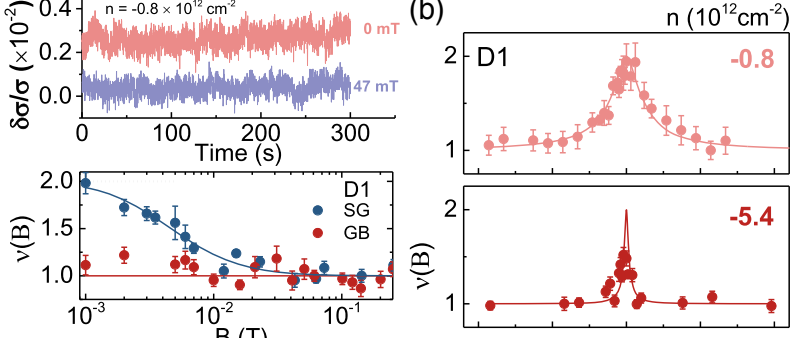

(d)
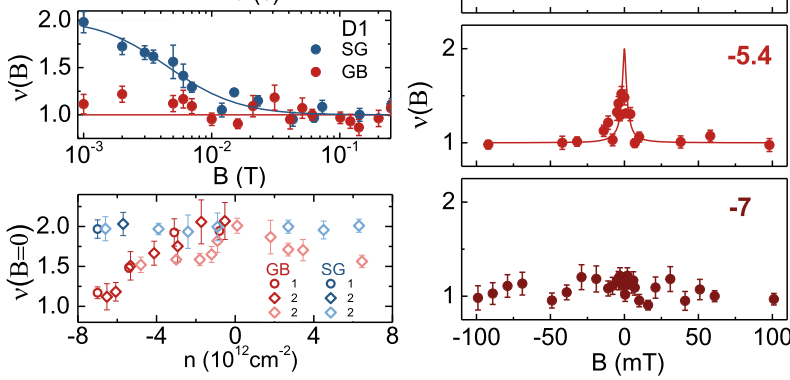

FIG. 3. (a) Conductivity fluctuations for GB region of D1 at $n=-0.8 \times 10^{12} \mathrm{~cm}^{-2}$ for $B=0 \mathrm{mT}$ (pink) and $B=47 \mathrm{mT}$ (purple), clearly indicating a reduction in the fluctuation magnitude at $B \gg B_{\phi}$. (b) $\nu(B)$ plotted for three different $n$ at $T=0.3 \mathrm{~K}$ for D1 showing spontaneous TRS breaking at zero field as $n$ is increased. Solid lines are fits to Eq. (1). (c) $\nu(B)$ for the SG and GB regions plotted at $T=0.3 \mathrm{~K}$ for D1 at $n=$ $-7 \times 10^{12} \mathrm{~cm}^{-2}$ indicating that spontaneous TRS breaking occurs only in the presence of a GB. (d) Noise reduction factor $\nu(B=0)$ for the SG and GB regions of D1 (circles) measured at $T=0.3 \mathrm{~K}$ (darker) and $\mathrm{D} 2$ (diamonds) at $T=0.3 \mathrm{~K}$ (darker) and $T=4.5 \mathrm{~K}$ (lighter) as a function of $n$.

GB regions at low $n$, similar to $2 \mathrm{D}$ systems such as exfoliated graphene [37], topological insulators [50], doped $\mathrm{Si} / \mathrm{Ge}$ systems [51], and nonmagnetic films [47]. However, with increasing $n$, a progressive reduction in $\nu(B)$ at $B=0$ was observed across the GB approaching unity, and thus $B$-independent $\nu$, for $n \gtrsim-6 \times 10^{12} \mathrm{~cm}^{-2}$ [Fig. 3(b), bottom panel]. The insensitivity of $\nu(B)$ to transverse field at the scale $B \sim B_{\phi}$ is a unique characteristic of systems with spontaneously broken TRS, as observed before in ferromagnetic films [36] and lightly doped semiconductors in the strongly interacting regime [49]. A similar trend was observed for D2 [Figs. 3(d), S5, and S6] where the reduction in $\nu(B)$ was observed in both doping regimes. Remarkably, the spontaneous breaking of TRS was observed only in the intergrain region, while the intragrain region continues to show a factor-of- 2 reduction in UCF magnitude with $B$ at similar high densities [Fig. 3(c), higher $T$ in Fig. S9]. The near $B$ independence of $\nu$ at high $n$ was found to be ubiquitous to quantum transport across GBs in CVD graphene, as shown for D1 in Fig. 3(b) and D2 in Fig. S5. The solid lines in Figs. 3(b) and 3(c) correspond to fits of $\nu(B)$ according to Eq. (1), with $l_{\phi}$ as the only fitting parameter. Figure 3(d) shows the noise reduction factor $\nu(B=0)=N(B=0) / N_{\phi}$ for D1 and D2 measured at $T=0.3$ and $4.5 \mathrm{~K}$ as a function of $n$. At $T=4.5 \mathrm{~K}$, the minimum $\nu(B)(\approx 1.5)$ at highest experimental $n$ indicate only partial removal of TRS. The factor-of- 2 reduction of $\nu(B)$ across the SG region was maintained throughout the 


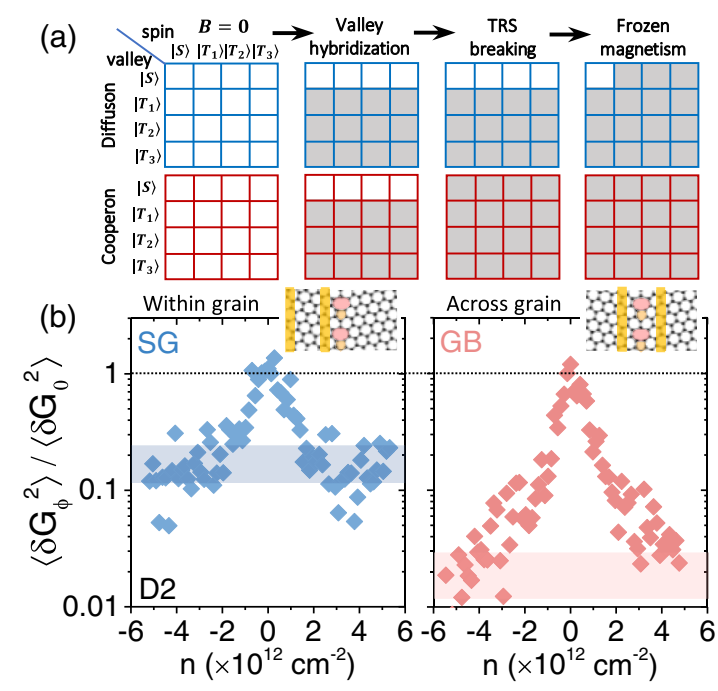

FIG. 4. (a) Schematic describing the contribution of diffuson (blue) and cooperon (red) singlet $(|S\rangle)$ and triplet $\left(\left|T_{1}\right\rangle,\left|T_{2}\right\rangle\right.$ and $\left|T_{3}\right\rangle$ ) states to the UCF magnitude $\left\langle\delta G_{\phi}^{2}\right\rangle$ in different symmetry classes. Valley hybridization leads to a factor of 4 reduction in $\left\langle\delta G_{\phi}^{2}\right\rangle$, while magnetic impurities reduce $\left\langle\delta G_{\phi}^{2}\right\rangle$ by a further factor of 8 due to gapping of spin diffuson triplets and all cooperons. (b) The variance in conductance $\left\langle\delta G_{\phi}^{2}\right\rangle$ within a phase-coherent box of $l_{\phi}^{2}$ normalized to its value at the Dirac point $\delta\left\langle G_{0}^{2}\right\rangle$ as a function of $n$ at $T=0.3 \mathrm{~K}$, showing a factor of $\approx 4$ reduction in the $\mathrm{SG}$ region and a factor of $\approx 30$ reduction in the GB region.

entire density range, implying that TRS is lifted solely in the presence of the GB.

To understand the origin of TRS breaking in the GB region, we then measured the $n$ dependence of the zero- $B$ magnitude of the UCF, which can distinguish between TRS breaking from an external $B$ field and that from an emergent frozen magnetic state [52]. For this, $\left\langle\delta G_{\phi}^{2}\right\rangle$ was calculated from reproducible fluctuations in $G$ within small windows of $V_{\mathrm{BG}}$ i.e., from $E_{\mathrm{F}}(\mathrm{SM} \mathrm{Sec}$. S5). The $\mathrm{SG}$ region exhibits a factor of $\approx 4$ reduction in $\left\langle\delta G_{\phi}^{2}\right\rangle$ [Fig. 4(b), left panel) due to valley symmetry lifting, thereby suppressing the UCF from valley triplet channels, a behavior observed in exfoliated graphene [37]. In contrast, the UCF magnitude in the GB region exhibits a drastic reduction [Fig. 4(b), right panel] by a factor of $\approx 30$ as $n$ is increased. This unique and unprecedented reduction can be quantitatively understood from a combination of valley hybridization, TRS breaking, and suppression of spin triplet channels in the presence of static (measurement time short compared to Korringa relaxation time) spin-dependent scattering, as depicted schematically in Fig. 4(a). The static spin texture, or "frozen magnetic state," at large $n$ may happen when the defect-bound magnetic impurities interact via RudermanKittel-Kasuya-Yosida (RKKY) exchange, forming long or short (e.g., a spin glass) range spin-ordered states [46,53]. Thus, the UCF measurement in graphene containing a GB suggests a rather unexpected effect of doping, which
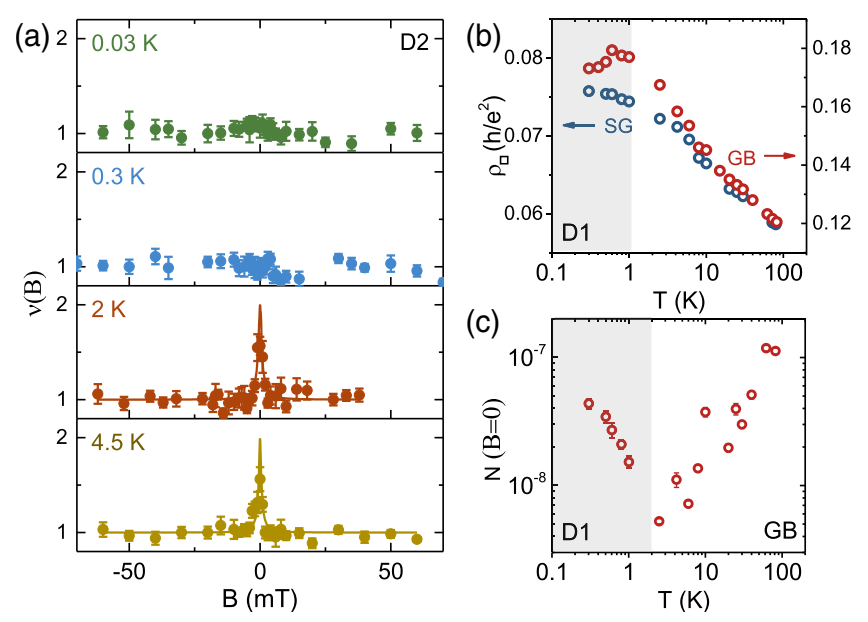

FIG. 5. (a) $\nu(B)$ plotted for D2 at $n=6.1 \times 10^{12} \mathrm{~cm}^{-2}$ showing spontaneous TRS breaking at zero field only at temperatures $T \lesssim 2 \mathrm{~K}$. (b) $T$ dependence of the reduced sheet resistance $\rho_{\square}$ (in units of $h / e^{2}$ ) averaged from resistance fluctuations measurements at $B=0 \mathrm{~T}$ for $\mathrm{SG}$ (blue) and $\mathrm{GB}$ (red) regions at $n=$ $-6 \times 10^{12} \mathrm{~cm}^{-2}$ for D1. (c) Normalized variance $N=S_{\sigma} / \sigma^{2}$ at $B=0$ as a function of temperature is plotted for GB region of D1 at $n=-6.9 \times 10^{12} \mathrm{~cm}^{-2}$, clearly indicating a sharp increase in $N(B=0)$ at lower temperatures.

manifests in both valley and (static) spin polarization when the carrier density is made sufficiently large.

To estimate the energy scale for local moment interaction at the GB, we study the effect of temperature on $\nu(B)$. The normalized magnetonoise $\nu(B)$ for the GB region in device D2 at $n=6.1 \times 10^{12} \mathrm{~cm}^{-2}$ with varying temperature is shown in Fig. 5(a) [data at higher $T$ in Fig. S7(a)]. Evidently, the spontaneous TRS breaking occurs only at temperatures $\lesssim 2 \mathrm{~K}$, while $\nu(B)$ approaches $\sim 2$ as $T$ is increased (data for D2 at $T=4.5 \mathrm{~K}$ in Fig. S6 and D3 at $T=8 \mathrm{~K}$ in Fig. S8). To estimate the exchange interaction between moments, we first estimate the Kondo temperature $T_{\mathrm{K}} \simeq 20 \mathrm{~K}$ from the $T$ dependence of the sheet resistance $R_{\square}$ at finite $B$ where WL corrections are suppressed (Fig. S14). This $T_{\mathrm{K}}$ is compatible with studies on irradiated graphene [3,4]. The RKKY interaction between moments can be estimated as [54] $k_{B} T_{\mathrm{RKKY}} \sim$ $27 j^{2} a^{4} / 64 \pi v_{F} \hbar R^{3} \sim 2.2 \mathrm{~K}$ (lattice constant $a \approx 0.246 \mathrm{~nm}$ and Fermi velocity in graphene $v_{F} \approx 10^{6} \mathrm{~m} \mathrm{~s}^{-1}$ ), where the Kondo exchange $j \approx 2.3 \mathrm{eV}$ was obtained from the experimental $T_{\mathrm{K}}$ and the DOS in the GB region, $D\left(E_{F}\right) \sim$ $0.05 \mathrm{eV}^{-1}$ (Fig. S12) [5-7]. Such a large $j$ value agrees with previous theoretical calculations [55-57]. The average defect distance $R \approx 2 \mathrm{~nm}$ can be estimated from the HRTEM image of the GB region on alignment in twobeam condition [SM Fig. S13(a) with corresponding histogram in Fig. S13(b)]. This value of $T_{\text {RKKY }}$ agrees reasonably well with the $T$ dependence of $\nu(B=0)$ [Fig. S7(b)], showing a continual increase in $\nu(B=0)$ up to $T \approx 10 \mathrm{~K}$, after which the decrease in $\nu(B=0)$ can 
be attributed to the loss of phase coherence through thermal averaging [Fig. S7(a)]. The estimated values of $T_{\mathrm{K}}$ and $T_{\mathrm{RKKY}}$ signal a competition between Kondo singlet formation and a frozen magnetic state [58]. To gain further insight into the nature of this magnetic state, we have measured the time-averaged resistivity $\rho_{\square}$ at $n=$ $-6 \times 10^{12} \mathrm{~cm}^{-2}$ for the GB and SG regions of D1 simultaneously. A distinctive feature of the $T$ dependence of $\rho_{\square}$ in the GB is a noticeable downturn at $T \lesssim 1 \mathrm{~K}$ [Fig. 5(b)], unlike the SG resistivity, despite accounting for quantum interference and $e-e$ interaction corrections. Such a resistivity downturn at low $T$ is strongly indicative of spin-glass freezing resulting from reduced spin-flip scattering $[59,60]$. Additionally, the normalized variance $N(B=0)$ of the GB region increases rapidly by nearly an order of magnitude on cooling from $\sim 2.5 \mathrm{~K}$ to $0.3 \mathrm{~K}$ [Fig. 5(c)] despite the low- $T$ saturation in $\gamma_{\mathrm{GB}}$ [Fig. 2(e)], contrasting with the behavior of SG noise (Fig. S15). Such an anomalous increase cannot be explained by the standard Feng-Lee-Stone theory [48] but can be attributed to the chaotic nature of spin reorganization below the spinglass freezing temperature [61], as previously reported in CuMn [62,63], AuFe [64,65], and dilute magnetic semiconductors [66].

In conclusion, we have identified signatures of spontaneous TRS breaking at graphene GBs using quantum transport measurements of WL and UCF. Such states emerge at high densities and at temperatures below $\sim 2 \mathrm{~K}$, suggesting low-energy spin-spin interactions, possibly mediated by RKKY coupling. An anomalously sharp increase in noise below $\sim 2 \mathrm{~K}$ indicates that the TRS breaking is likely due to an emergent spin-glass state at graphene GBs.

We are grateful to H.R. Krishnamurthy, Sumilan Banerjee, and Sudipta Dutta for useful discussions. K. H., V. K., and A. G acknowledge the Department of Science and Technology (DST) for a funded project. K. H. and A. G. also thank the National Nanofabrication Center (NNfC) at the Centre for Nano Science and Engineering (CeNSE), Indian Institute of Science (IISc), for providing clean room facilities.

K. H. and V. K. contributed equally to this work.

Note added in the proof.-After the submission of this Letter, we became aware of a complementary work [67] using STM measurements to probe Yu-Shiba-Rusinov states on graphene grain boundaries. We also became aware of Ref. [68], which explores chiral symmetry breaking in Li-intercalated graphene using ARPES and STM measurements.

*Corresponding author.

kimberly@iisc.ac.in
${ }^{\dagger}$ Present address: Materials Science Centre, Indian Institute of Technology Kharagpur, Kharagpur 721302, India.

${ }^{\ddagger}$ Present address: Department of Physics, Arizona State University, Tempe, Arizona 85287, USA.

${ }^{\S}$ Present address: Department of Metallurgical and Material Engineering, Indian Institute of Technology Kharagpur, Kharagpur 721302, India.

[1] M. M. Ugeda, I. Brihuega, F. Guinea, and J. M. GómezRodríguez, Phys. Rev. Lett. 104, 096804 (2010).

[2] K. M. McCreary, A. G. Swartz, W. Han, J. Fabian, and R. K. Kawakami, Phys. Rev. Lett. 109, 186604 (2012).

[3] J.-H. Chen, L. Li, W. G. Cullen, E. D. Williams, and M. S. Fuhrer, Nat. Phys. 7, 535 (2011).

[4] Y. Jiang, P.-W. Lo, D. May, G. Li, G.-Y. Guo, F. B. Anders, T. Taniguchi, K. Watanabe, J. Mao, and E. Y. Andrei, Nat. Commun. 9, 2349 (2018).

[5] L. Kou, C. Tang, W. Guo, and C. Chen, ACS Nano 5, 1012 (2011).

[6] S. S. Alexandre, A. D. Lúcio, A. H. Castro Neto, and R. W. Nunes, Nano Lett. 12, 5097 (2012).

[7] S. Dutta and K. Wakabayashi, Sci. Rep. 5, 11744 (2015).

[8] P. Nemes-Incze, P. Vancsó, Z. Osváth, G. I. Márk, X. Jin, Y.-S. Kim, C. Hwang, P. Lambin, C. Chapelier, and L. PéterBiró, Carbon 64, 178 (2013).

[9] A. Luican-Mayer, J. E. Barrios-Vargas, J. T. Falkenberg, G. Autès, A. W. Cummings, D. Soriano, G. Li, M. Brandbyge, O. V. Yazyev, S. Roche, and E. Y. Andrei, 2D Mater. 3, 031005 (2016).

[10] Q. Yu, L. A. Jauregui, W. Wu, R. Colby, J. Tian, Z. Su, H. Cao, Z. Liu, D. Pandey, D. Wei, T. F. Chung, P. Peng, N. P. Guisinger, E. A. Stach, J. Bao, S.-S. Pei, and Y. P. Chen, Nat. Mater. 10, 443 (2011).

[11] F. Gargiulo and O. V. Yazyev, Nano Lett. 14, 250 (2014).

[12] O. V. Yazyev and S. G. Louie, Nat. Mater. 9, 806 (2010).

[13] D. Van Tuan, J. Kotakoski, T. Louvet, F. Ortmann, J. C. Meyer, and S. Roche, Nano Lett. 13, 1730 (2013).

[14] J. C. Koepke, J. D. Wood, D. Estrada, Z.-Y. Ong, K. T. He, E. Pop, and J. W. Lyding, ACS Nano 7, 75 (2013).

[15] K. W. Clark, X.-G. Zhang, I. V. Vlassiouk, G. He, R. M. Feenstra, and A.-P. Li, ACS Nano 7, 7956 (2013).

[16] L. Tapasztó, P. Nemes-Incze, G. Dobrik, K. Jae Yoo, C. Hwang, and L.P. Biró, Appl. Phys. Lett. 100, 053114 (2012).

[17] L. A. Jauregui, H. Cao, W. Wu, Q. Yu, and Y. P. Chen, Solid State Commun. 151, 1100 (2011).

[18] A. W. Tsen, L. Brown, M. P. Levendorf, F. Ghahari, P. Y. Huang, R. W. Havener, C. S. Ruiz-Vargas, D. A. Muller, P. Kim, and J. Park, Science 336, 1143 (2012).

[19] V. Kochat, C. S. Tiwary, T. Biswas, G. Ramalingam, K. Hsieh, K. Chattopadhyay, S. Raghavan, M. Jain, and A. Ghosh, Nano Lett. 16, 562 (2016).

[20] J. Lahiri, Y. Lin, P. Bozkurt, I. I. Oleynik, and M. Batzill, Nat. Nanotechnol. 5, 326 (2010).

[21] P. Y. Huang, C. S. Ruiz-Vargas, A. M. van der Zande, W. S. Whitney, M. P. Levendorf, J. W. Kevek, S. Garg, J. S. Alden, C. J. Hustedt, Y. Zhu, J. Park, P. L. McEuen, and D. A. Muller, Nature (London) 469, 389 (2011).

[22] J. Červenka, M. I. Katsnelson, and C. F. J. Flipse, Nat. Phys. 5, 840 (2009).

[23] C. W. J. Beenakker, Rev. Mod. Phys. 69, 731 (1997). 
[24] See Supplemental Material, which includes Refs. [25-34], at http://link.aps.org/supplemental/10.1103/PhysRevLett .126 .206803 for additional data.

[25] P. A. Lee, A. D. Stone, and H. Fukuyama, Phys. Rev. B 35, 1039 (1987).

[26] J. M. Soler, E. Artacho, J. D. Gale, A. García, J. Junquera, P. Ordejón, and D. Sánchez-Portal, J. Phys. Condens. Matter 14, 2745 (2002).

[27] N. Troullier and J. L. Martins, Phys. Rev. B 43, 1993 (1991).

[28] J. P. Perdew, K. Burke, and M. Ernzerhof, Phys. Rev. Lett. 77, 3865 (1996).

[29] A. Ghosh, S. Kar, A. Bid, and A. Raychaudhuri, arXiv: cond-mat/0402130.

[30] A. Trionfi, S. Lee, and D. Natelson, Phys. Rev. B 70, 041304(R) (2004).

[31] A. Cortijo and M. A. Vozmediano, Nucl. Phys. B763, 293 (2007).

[32] S. Lara-Avila, A. Tzalenchuk, S. Kubatkin, R. Yakimova, T. J. B. M. Janssen, K. Cedergren, T. Bergsten, and V. Falko, Phys. Rev. Lett. 107, 166602 (2011).

[33] J. Jobst, D. Waldmann, I. V. Gornyi, A. D. Mirlin, and H. B. Weber, Phys. Rev. Lett. 108, 106601 (2012).

[34] D. Goldhaber-Gordon, J. Göres, M. A. Kastner, H. Shtrikman, D. Mahalu, and U. Meirav, Phys. Rev. Lett. 81, 5225 (1998).

[35] A. D. Stone, Phys. Rev. B 39, 10736 (1989).

[36] S. Lee, A. Trionfi, and D. Natelson, Phys. Rev. B 70, 212407 (2004).

[37] A. N. Pal, V. Kochat, and A. Ghosh, Phys. Rev. Lett. 109, 196601 (2012).

[38] E. McCann, K. Kechedzhi, V. I. Falko, H. Suzuura, T. Ando, and B. L. Al'tshuler, Phys. Rev. Lett. 97, 146805 (2006).

[39] F. Pierre, H. Pothier, D. Esteève, M. H. Devoret, A. B. Gougam, and N. O. Birge, in Kondo Effect and Dephasing in Low-Dimensional Metallic Systems, edited by V. Chandrasekhar, C. Van Haesendonck, and A. Zawadowski (Kluwer, Dordrecht, 2001), pp. 119-132.

[40] F. Pierre and N. O. Birge, Phys. Rev. Lett. 89, 206804 (2002).

[41] F. Pierre, A. B. Gougam, A. Anthore, H. Pothier, D. Esteve, and N. O. Birge, Phys. Rev. B 68, 085413 (2003).

[42] S. V. Morozov, K. S. Novoselov, M. I. Katsnelson, F. Schedin, D. C. Elias, J. A. Jaszczak, and A. K. Geim, Phys. Rev. Lett. 100, 016602 (2008).

[43] F. V. Tikhonenko, A. A. Kozikov, A. K. Savchenko, and R. V. Gorbachev, Phys. Rev. Lett. 103, 226801 (2009).

[44] M. B. Maple, in Magnetism Volume V: Magnetic Properties of Magnetic Alloys, edited by H. Suhl (Academic Press, New York, 1973), p. 289.

[45] C. V. Haesendonck, J. Vranken, and Y. Bruynseraede, Phys. Rev. Lett. 58, 1968 (1987).
[46] F. Schopfer, C. Bäuerle, W. Rabaud, and L. Saminadayar, Phys. Rev. Lett. 90, 056801 (2003).

[47] N. O. Birge, B. Golding, and W. H. Haemmerle, Phys. Rev. B 42, 2735 (1990).

[48] S. Feng, P. A. Lee, and A. D. Stone, Phys. Rev. Lett. 56, 1960 (1986).

[49] S. Shamim, S. Mahapatra, G. Scappucci, W. M. Klesse, M. Y. Simmons, and A. Ghosh, Phys. Rev. Lett. 112, 236602 (2014).

[50] S. Islam, S. Bhattacharyya, H. Nhalil, S. Elizabeth, and A. Ghosh, Phys. Rev. B 97, 241412(R) (2018).

[51] S. Shamim, S. Mahapatra, G. Scappucci, W. Klesse, M. Simmons, and A. Ghosh, Sci. Rep. 7, 46670 (2017).

[52] E. Akkermans and G. Montambaux, Mesoscopic Physics of Electrons and Photons (Cambridge University Press, Cambridge, England, 2007).

[53] P. W. Anderson, in Les Houches, Ill-Condensed Matter, Session XXXI, edited by R. Balian, R. Maynard, and G. Toulouse (North Holland, Amsterdam, 1978), pp. 159-261.

[54] M. Sherafati and S. Satpathy, Phys. Rev. B 83, 165425 (2011).

[55] K. Sengupta and G. Baskaran, Phys. Rev. B 77, 045417 (2008).

[56] M. A. Cazalilla, A. Iucci, F. Guinea, and A. H. Castro Neto, arXiv:1207.3135.

[57] A. K. Mitchell and L. Fritz, Phys. Rev. B 88, 075104 (2013).

[58] S. Doniach, Physica (Amsterdam) 91B+C, 231 (1977).

[59] G. Neuttiens, J. Eom, C. Strunk, V. Chandrasekhar, C. V. Haesendonck, and Y. Bruynseraede, Europhys. Lett. 34, 617 (1996).

[60] G. Forestier, M. Solana, C. Naud, A. D. Wieck, F. m. c. Lefloch, R. Whitney, D. Carpentier, L. P. Lévy, and L. Saminadayar, Phys. Rev. B 102, 024206 (2020).

[61] S. Feng, A. J. Bray, P. A. Lee, and M. A. Moore, Phys. Rev. B 36, 5624 (1987).

[62] N. E. Israeloff, M. B. Weissman, G. J. Nieuwenhuys, and J. Kosiorowska, Phys. Rev. Lett. 63, 794 (1989).

[63] P. W. Fenimore and M. B. Weissman, J. Appl. Phys. 85, 8317 (1999).

[64] K. A. Meyer and M. B. Weissman, Phys. Rev. B 51, 8221 (1995).

[65] G. Neuttiens, C. Strunk, C. Van Haesendonck, and Y. Bruynseraede, Phys. Rev. B 62, 3905 (2000).

[66] J. Jaroszyński, J. Wróbel, G. Karczewski, T. Wojtowicz, and T. Dietl, Phys. Rev. Lett. 80, 5635 (1998).

[67] E. Cortés-del Río, J. L. Lado, V. Cherkez, P. Mallet, J.-Y. Veuillen, J. C. Cuevas, J. M. Gómez-Rodríguez, J. Fernández-Rossier, I. Brihuega, arXiv:2010.10377.

[68] C. Baoet al., following Letter, Phys. Rev. Lett. 126, 206804 (2021). 